In Crescendo, 2019; 10(1): 215-221

Fecha de recepción: 16 de diciembre del 2018

Fecha de aceptación: 22 de marzo del 2019

\title{
CALIDAD DEL SERVICIO Y SATISFACCIÓN DEL CLIENTE EN LAS MYPE DEL SECTOR SERVICIOS - RUBRO HOTELES DE 3 ESTRELLAS DEL DISTRITO DE PIURA, 2015
}

\author{
QUALITY OF SERVICE AND CUSTOMER SATISFACTION IN \\ THE SERVICES SECTOR MSE - ITEM 3 STAR HOTELS \\ DISTRICT PIURA, 2015
}

Carlos Eduardo Arias-Muñoz ${ }^{1}$

\section{RESUMEN}

El presente trabajo de investigación tuvo como objetivo general determinar la influencia de la Calidad del Servicio en la Satisfacción de los clientes en las micro y pequeñas empresas del sector servicios - rubro Hoteles de 3 Estrellas del distrito de Piura, 2015. Para el desarrollo del trabajo se utilizó un tipo y nivel de investigación correlacional y un diseño no experimentaltransaccional. Para el recojo de la información se identificó una población de 329 clientes, de los cuales se escogió una muestra de 141 clientes a quienes se les aplicó un cuestionario de 44 preguntas cerradas por medio de la encuesta; de lo cual se obtuvo los siguientes resultados: El 53,9\% de clientes encuestados, expresan estar algo de acuerdo en cuanto a la modernidad del mobiliario, equipos y herramientas, el 43,3\% manifiesta estar apenas de acuerdo respecto a la entrega del servicio con la menor cantidad de errores, el 46,8\% manifiesta estar algo de acuerdo respecto al comportamiento de los empleados en la inspiración de confianza y el $50.4 \%$ considera estar apenas de acuerdo que los empleados tienen conocimientos suficientes para responder preguntas. Finalmente, se concluye que la calidad del servicio que aplican los hoteles de 3 estrellas del distrito de Piura incide desfavorablemente en el logro de la satisfacción de sus clientes.

PALABRAS CLAVE: MYPE, calidad de servicio y hotel.

1 Licenciado en Administración de Empresas, Docente Tutor de la Escuela de Administración de la Universidad Católica Los Ángeles de Chimbote - ULADECH - Centro Académico Sullana - Escuela Profesional de Administración. Dirección Electrónica: cariasmunoz@hotmail.com 


\begin{abstract}
The present research had as general objective to determine the influence of Service Quality in Customer Satisfaction in the micro and small enterprises in the services sector - category $3 *$ hotels district of Piura, 2015. Work for the development of a type and level of correlational research and a non - transactional experimental design was used. To gather information on a population of 329 customers, of which a sample of 141 customers who were applied a questionnaire of 44 questions closed by the survey was chosen was identified; of which the following results were: $53.9 \%$ of surveyed customers expressed to be some agreement as to the modernity of furniture, equipment and tools , $43.3 \%$ claim to be just agreed on the delivery of service with fewer errors, the $46.8 \%$ claim to be some agreement on the behavior of employees in inspiring confidence and $50.4 \%$ felt they just agree that employees have sufficient knowledge to answer questions. Finally in conclusion: The quality of service applied by 3 star hotels Piura district adverseley impact on achieving customer.
\end{abstract}

KEY WORDS: MYPE, quality of service and hotel.

\title{
INTRODUCCIÓN
}

En el distrito de Piura las MYPE del sector servicios - rubro hoteles pocas son las empresas que gestionan estándares de calidad para brindar un buen servicio en comparación con otras ciudades del país. El servicio de hospedaje que brindan las MYPE es medianamente regular, si bien es cierto tienen mucho más interés en mejorar su infraestructura y equipamiento no se preocupan en mejorar la calidad de sus servicios como el de invertir en capacitación de su personal, porque siendo el personal de contacto quien brinda un servicio deficiente al cliente por falta de experiencia, capacitación, etc. y por ser un factor de suma importancia que el cliente evalúa, es un factor clave que limita una buena gestión de calidad. Los únicos criterios que realmente cuentan en la evaluación de la calidad de un servicio son los que establecen los clientes. Solo los usuarios juzgan la calidad (Zeithaml, Parasuraman, \& Berry, 2007).

Son escasas las empresas hoteleras que se dedican a diseñar estrategias para incrementar sus estándares de calidad, este sector empresarial en Piura está desfasado en comparación con el resto del Perú. El servicio que se brinda a los clientes en la ciudad es medianamente regular (Adriazén, 2011). La gestión de la calidad se convierte en condición necesaria para cualquier estrategia dirigida al éxito de las empresas (Gadotti \& Franca, 2008). 
Entonces, de continuar con este nivel de servicio, los perjudicados serán los empresarios, porque si no dan la calidad del servicio requerido, el cliente se sentirá insatisfecho; los huéspedes podrían dar preferencia a otras ciudades del país o su permanencia sería solo lo indispensable de ser el caso, lo cual originará que los ingresos y rentabilidad de estas empresas disminuya, y al largo plazo tengan que desaparecer. La micro y pequeñas empresas con su espíritu emprendedor, con sus posibilidades de expansión y mayor competitividad, tendrán que brindar un mejor servicio para así tener una buena posición en el mercado. La satisfacción del cliente deberá ser estudiada de forma continua, por tratarse de una variable que constituye la principal fuente para crear lealtad, retener clientes y definir estrategias acordes con un mercado que cada vez es más exigente y cambiante (Pontón, 2009).

Por lo anteriormente expresado, el enunciado del problema general de investigación es el siguiente: ¿De qué manera la Calidad del Servicio influye en la Satisfacción del Cliente en las micro y pequeñas empresas del sector servicios rubro Hoteles de 3 Estrellas del distrito de Piura, 2015?

Para dar respuesta al problema se propuso como objetivo general: Determinar la influencia de la Calidad del Servicio en la Satisfacción de los clientes en las micro y pequeñas empresas del sector servicios - rubro Hoteles de 3 Estrellas del distrito de Piura, 2015 y como objetivos específicos: Determinar el nivel de calidad del servicio en las micro y pequeñas empresas del sector servicios - rubro Hoteles de 3 Estrellas del distrito de Piura, 2015 y determinar el nivel de Satisfacción del Cliente en las micro y pequeñas empresas del sector servicios - rubro Hoteles de 3 Estrellas del distrito de Piura, 2015. La hipótesis general de la investigación es: la calidad del servicio de la micro y pequeñas empresas del Sector Servicios Rubro Hoteles de 3 Estrellas del distrito de Piura incide desfavorablemente en la satisfacción del cliente.

Finalmente la investigación se justificó, por ser necesario conocer si se está gestionando la calidad de servicio de manera eficiente en las MYPE a través de sus características como aspectos tangibles, confiabilidad, capacidad de respuesta, seguridad y empatía las mismas que permiten a los clientes sentir satisfacción por el servicio recibido, de tal manera que los clientes no tengan quejas ni reclamos en la atención que se le brinda, logrando una buena percepción del servicio en las micro y pequeñas empresas del sector servicios - rubro Hoteles de 3 Estrellas del distrito de Piura, 2015. 


\section{MÉTODOS}

La población para este estudio fueron los clientes o huéspedes que se alojaron en las 4 MYPE del sector servicio - rubro hoteles de 3 estrellas del distrito de Piura con registro REMYPE (Registro Nacional de la Micro y Pequeña Empresa) del Ministerio de Trabajo y Promoción del Empleo, hallándose una muestra de 141 personas. Como Técnicas de recolección de datos se consideró la encuesta, y el instrumento de recolección de datos fue el cuestionario estructurado.

En cuanto a la metodología utilizada en la presente investigación fue del Tipo correlacional ya que se relacionó las variables Calidad del Servicio y Satisfacción de los clientes de las MYPE del sector servicios - rubro hoteles de 3 estrellas del distrito de Piura, 2015. En cuanto al nivel de la investigación fue cuantitativa, el diseño de la investigación fue no experimental porque se observan los fenómenos tal cual se dan en su estado natural; así mismo es transaccional o transversal, porque se recolectó los datos en un solo momento, en un tiempo único.

Los datos obtenidos fueron incorporados a los programas especiales de cómputo, MS Excel, SPSS versión 20 demuestra el grado confiabilidad de las variables; Para el análisis de la información se presentó en cuadros y gráficos. Asimismo, se recurrió a la prueba no paramétrica chi - cuadrado, que mediante las tablas cruzadas de las variables se obtiene si existe o no esta incidencia o influencia. (Prueba de la independencia), para la contrastación de la hipótesis.

En el presente trabajo se hizo hincapié a los principios éticos de confidencialidad, respeto a la dignidad de la persona y respeto a la propiedad intelectual, asimismo se reconoce que toda información utilizada en el presente trabajo ha sido con fines exclusivamente académicos.

\section{RESULTADOS}

Se encontró un chi cuadrado de 403,346, siendo -con un grado de libertad igual a veinticinco-mayor de 37,7 , significa que

$$
X_{0}^{2}>X_{t}^{2}
$$

entonces, se acepta la hipótesis general; por lo tanto, incide desfavorablemente en la Satisfacción del Cliente. 
Tabla 1

DISTRIBUCIÓN PROMEDIO DE LOS CINCO CRITERIOS DE LA CALIDAD

DEL SERVICIO EN LAS MICRO Y PEQUEÑAS EMPRESAS DEL SECTOR

SERVICIOS - RUBRO HOTELES DE 3 ESTRELLAS DEL DISTRITO

DE PIURA, 2015

\begin{tabular}{|c|c|c|c|c|c|}
\hline & Elementos tangibles & Fiabilidad & Capacidad de respuesta & Seguridad & Empatía \\
\hline Promedio & 5,76 & 5,45 & 5,56 & 5,57 & 5,53 \\
\hline \multicolumn{2}{|c|}{ Promedio general } & \multicolumn{2}{|c|}{5,58} & \multicolumn{2}{c|}{$79,71 \%$} \\
\hline
\end{tabular}

Nota: Elaboración propia.

Interpretación: En la Tabla 01, se puede observar que el promedio general es de 5,58 y el índice general es de 79,71\% que en el rango de porcentaje del 0-100 en la escala de Likert es: "Algo de acuerdo".

Tabla 2

DISTRIBUCIÓN PROMEDIO DE SATISFACCIONES DE LAS DIMENSIONES

EVALUADAS EN LAS MICRO Y PEQUEÑAS EMPRESAS DEL SECTOR SERVICIOS - RUBRO HOTELES DE 3 ESTRELLAS DEL

DISTRITO DE PIURA, 2015

\begin{tabular}{|c|c|c|c|c|c|}
\hline & Elementos tangibles & Fiabilidad & Capacidad de respuesta & Seguridad & Empatía \\
\hline Promedio & 5,80 & 5,62 & 5,62 & 5,69 & 5,70 \\
\hline \multicolumn{2}{|c|}{ Promedio general } & \multicolumn{2}{|c|}{5,69} & \multicolumn{2}{c|}{$81,29 \%$} \\
\hline
\end{tabular}

Nota: Elaboración propia.

Interpretación: En la Tabla 02, se puede observar que el promedio general es de 5,69 y el índice general es de 81,29\% que en el rango de porcentaje del 0-100 en la escala de Likert es: "Algo de acuerdo".

\section{DISCUSIÓN}

De acuerdo a lo planteado por Alén \& Fraiz, (2006), el modelo estructural completo en el que se plasman las relaciones causales entre los constructos calidad de servicio y satisfacción... "Se encontró que la calidad de servicio es un antecedente importante de la satisfacción, sobre la que ejerce una influencia clara y significativa (0.740), quedando de este modo contrastada positivamente la hipótesis $1 a^{\prime \prime}$. 
La aplicación de este modelo, es similar a los resultados obtenidos en la presente investigación, porque la calidad del servicio que aplican las micro y pequeñas empresas del Sector Servicios - Rubro Hoteles de 3 Estrellas del distrito de Piura, inciden en la satisfacción de sus clientes, lo cual fue posible deliberar mediante la prueba estadística a la que fue sometida.

De acuerdo a lo planteado por Alén \& Fraiz, (2006), el modelo estructural completo en el que se plasman las relaciones causales entre los constructos calidad de servicio y satisfacción..."Por otra parte, desde un punto de vista académico, la utilización de modelos de ecuaciones estructurales con múltiples indicadores, nos ha permitido conocer mejor las influencias entre la calidad y la satisfacción en un modelo global, ya que todas las dimensiones de calidad de servicio ejercen una influencia positiva sobre la satisfacción".

La aplicación de este modelo, es similar a los resultados obtenidos en la presente investigación, porque la calidad del servicio que aplican las micro y pequeñas empresas del Sector Servicios - Rubro Hoteles de 3 Estrellas del distrito de Piura, 2015 inciden en la satisfacción de sus clientes, lo cual fue posible deliberar mediante la prueba estadística a la que fue sometida.

La gestión de la calidad en las micro y pequeñas empresas del Sector Servicios - Rubro Hoteles de 3 Estrellas del distrito de Piura, permitirá solucionar la problemática de las MYPE y obtener un óptimo nivel de estándares de calidad para brindar un buen servicio en comparación con otras ciudades del país. Con lo que se logrará un alto nivel de satisfacción de los clientes por el servicio recibido, esto se evidenciará con una oportuna capacitación al personal, para una adecuada atención de los huéspedes y a la mejora de la calidad de los servicios, que se transformará en el aumento de la satisfacción de los clientes y por ende en la economía de este sector, de la región y en la productividad del país en general.

\section{CONCLUSIONES}

- La calidad del servicio que aplican las micro y pequeñas empresas del Sector Servicios - Rubro Hoteles de 3 Estrellas del distrito de Piura, 2015 influye desfavorablemente en el logro de la satisfacción de sus clientes, sin embargo a medida que estas estrategias se apliquen deficientemente en la mayoría de sus dimensiones (tangibilidad, confiabilidad, respuesta, seguridad y empatía), da lugar a una menor incidencia en la satisfacción de los clientes de acuerdo a la relación directa comprobada entre ambas variables de investigación, que significa una desventaja para al rubro hoteles de 3 estrellas en estudio. 
- El índice de calidad percibida del servicio que brindan las micro y pequeñas empresas del Sector Servicios - Rubro Hoteles de 3 Estrellas del distrito de Piura, 2015 se ubica en el rango "algo de acuerdo" con un 79,71\%.

- El índice de satisfacción del servicio que brindan las micro y pequeñas empresas del Sector Servicios - Rubro Hoteles de 3 Estrellas del distrito de Piura, 2015 se ubica en el rango "algo de acuerdo" con un $81,29 \%$.

\section{REFERENCIAS BIBLIOGRÁFICAS}

Adriazén, V. (2011). Diseño de Operaciones y Determinación de los Indicadores de Calidad del Hotel Río Verde. (págs. v, 89-91).

Alén, M., \& Fraiz, J. (2006). Relación entre La Calidad de Servicio y La Satisfacción del Consumidor. Su Evaluación en el Ámbito del Turismo Termal. En A. E. Empresa (Ed.)., 12, págs. 253, $251-272$.

Gadotti dos Anjos, S. J., \& Franca de Abreu, A. (2008). Fundación Dialnet - Universidad de la Rioja.

Pontón, H. (2009). COEPTUM - Revista Electrónica de Gerencia Empresarial.

Zeithaml, V. A., Parasuraman, A., \& Berry, L. L. (2007). Calidad Total en la Gestión de Servicios. Madrid, España: Diaz de Santos, S. A. 